\title{
¿INFLUYEN LOS ESTILOS PARENTALES Y LA INTELIGENCIA EMOCIONAL DE LOS PADRES EN EL DESARROLLO EMOCIONAL DE SUS HIJOS ESCOLARIZADOS EN 2० CICLO DE EDUCACIÓN INFANTIL?
}

\author{
DO PARENTAL STYLES AND PARENTS' \\ EMOTIONAL INTELLIGENCE INFLUENCE \\ THEIR CHILDREN'S EMOTIONAL \\ DEVELOPMENT IN KINDERGARTEN SCHOOL?
}

\author{
Ana Ramírez-Lucas ${ }^{1}$, Mercedes Ferrando ${ }^{1}$ y Marta SÁinz GómeZ ${ }^{1}$
}

\begin{abstract}
Cómo referenciar este artículo/How to reference this article:
Ramírez-Lucas, A., Ferrando, M. y Sáinz Gómez, M. (2015). Influyen los estilos parentales y la Inteligencia Emocional de los padres en el desarrollo emocionaL de sus hijos escolarizados en $2^{\circ}$ ciclo de educación infantil? [Do parental styles and parents' emotional intelligence influence their children's emotional development in kindergarten school?]. Acción Psicológica, 12(1), 65-78. doi: http://dx.doi.org/10.5944/ap.12.1.14314
\end{abstract}

\section{Resumen}

La inteligencia emocional se ha relacionado con varias facetas del éxito personal y social como son el bienestar psicológico, el rendimiento académico entre otras. Las emociones y su regulación se aprenden desde la infancia y están influenciadas por el contexto más próximo. El presente trabajo tienen por objetivo estudiar la relación entre los estilos parentales e Inteligencia emocional de los padres y la IE de sus hijos/as en edades tempranas. Han participado un total de 83 niños escolarizados en $2^{\circ}$ ciclo de educación infantil; $52 \mathrm{pa}-$ dres y 62 madres. Se han utilizado como medida de la
IE de los niños: (a) el Cuestionario de Bar-On y Parker (2002) para observadores que mide las habilidades interpersonales, intrapersonales, manejo del estrés, adaptabilidad y estado de ánimo; (b) Prueba de Percepción y Valoración de las emociones (PERCERVAL, Mestre, et al., 2011). Los adultos cumplimentaron un (a) cuestionario sobre su propia Inteligencia emocional adaptado de Bar-On (1997) y (b) un cuestionario sobre sus estilos parentales adaptado de Robinson, Mandleco, Olsen y Hart (1995) el cual mide la intensidad de dos estilos parentales: el democrático y el autoritario. Los resultados mostraron que el estilo parental se relaciona con la Inteligencia emocional de los padres. Los padres/madres con mayor inteligencia emocional tienden a mostrar un

Correspondencia: Ana Ramírez-Lucas. Universidad de Murcia. Email: ana.ramirez.lucas@gmail.com

${ }^{1}$ Universidad de Murcia.

Recibido: 24 de marzo de 2015

Aceptado: 12 de mayo de 2015 
estilo democrático. A su vez, el estilo democrático de los padres/madres correlaciona en mayor medida con la IE de los niños. Se encontró además que dependiendo del estilo parental, los alumnos tienden a desarrollar más unas habilidades emocionales u otras, así, los niños con madres autoritarias tenían mayores habilidades interpersonales.

Palabras clave: Inteligencia emocional; PERCERVAL; estilos parentales.

\section{Abstract}

Emotional intelligence has been related to several facets of personal and social success as are psychological well-being, academic performance among others. Emotions and its regulation are learned during childhood and that learning is affected by the closest context. The present study aims to study the relationship between parents' emotional intelligence and parental styles and their children emotional intelligence in early ages. A total of 83 students, 52 parents and 62 mothers took part in this research. As measure of children emotional intelligence two instruments were used: (a) the questionnaire of Emotional Quotient designed by Bar-On and Parker (2002) to be completed by an observer (parents). This questionnaire asses the following abilities: interpersonal, intrapersonal, stress management, adaptability and general mood; and (b) Test of Perception and Assessment of Emotions (PERCERVAL, Mestre et al., 2011). Adults took two questionnaires: (a) an adaptation of Bar-On (1997) questionnaire to assess their own emotional intelligence; and (b) an adaptation of the Robinson et al (1995) questionnaire about parenting styles. This questionnaire assesses both: democratic and authoritarian styles. The obtained results point out that parents' parental style is correlated with parents' emotional intelligence. Parents'/ mothers' with higher emotional intelligence tend to show a democratic parental style. In addition, a democratic style of parents'/mothers' is correlated with a higher emotional intelligence of their children. Differences in emotional abilities were found deepening on the parental style; thus, children with authoritarian mothers tend to develop higher intrapersonal abilities.

Keywords: Emotional intelligence; PERCERVAL; parental styles.

\section{Introducción}

Durante los primeros años de vida resulta prácticamente imposible separar el desarrollo afectivo y el desarrollo social, pues ambos van de la mano y, en gran medida, son los responsables de la adecuada evolución en el resto de ámbitos del desarrollo infantil. Por lo que "una sólida base afectiva posibilita al niño abrirse al mundo físico y social con seguridad y confianza, actuando dicha base como facilitadora de una adecuada evolución cognitiva y psicosocial" (Ferrándiz, Fernández y Bermejo, 2011, p. 129).

En este sentido, la familia se presenta como la unidad básica de la sociedad (Fúlquez, 2011) y el primer núcleo social de convivencia para el ser humano. Es en el contexto familiar donde los niños aprenden de sus padres su base de conocimiento emocional, así como la competencia en la identificación y regulación emocional (Mayer y Salovey, 1997). Los padres, tienen un papel clave en el desarrollo de aptitudes sirviendo de modelo de sus hijos, pues las interacciones emocionales con el cuidador, además de fomentar el desarrollo emocional del niño, influyen en la maduración de las partes del cerebro encargadas de la conciencia y regulación emocional (Schoree, 1996).

En esta línea, son muchos los autores (JiménezMorales y López-Zafra, 2009; León-Rodríguez y Sierra, 2008) que coinciden en resaltar que la familia y la escuela son ámbitos imprescindibles en el desarrollo del ser humano debido a que existe una relación intensa entre el desarrollo de la comprensión de las consecuencias y la regulación emocional. Goleman (1996) apunta que la vida familiar es la cuna en la que el individuo comienza a sentirse él mismo y en donde se aprende la forma en la que los otros reaccionan ante los sentimientos. Es el espacio donde se aprende a pensar en sí mismo, en los propios sentimientos y en las posibles respuestas ante determinadas situaciones. En estas circunstancias es condición imprescindible que el niño posea figuras referenciales para avanzar óptimamente en su socialización. La familia es por tanto, "nuestra primera escuela para la alfabetización emocional y el contexto más importante donde se forjan las competencias emocionales" (Sánchez Nuñez, 2008, p.49).

En 1998, Eisenberg, Spinrad y Cumberland confeccionaron un modelo heurístico explicativo acerca de las vías por medio de las cuales los padres socializan emocionalmente a los hijos. Estas son: 1) las reacciones de los padres a las emociones de sus hijos, 2) la discusión de los padres sobre las emociones, 3) la expresión de las emociones de los padres y 4) la selección o modificación por los padres de situaciones propiciadoras de emociones (Sánchez Nuñez, 2008). 
$\mathrm{Si}$ atendemos a este modelo, se desprende que la función emocional de una familia se define por experiencias favorecedoras de mecanismos introspectivos, según los cuales, el sujeto goza de circunstancias que le enseñan progresivamente a afrontar y contener ansiedades, identificar sentimientos y problemas, con la finalidad de gestionar la reparación y simbolización (Fúlquez, 2011, p.88). Estos mecanismos se pueden agrupar en dos tipos de vías en función del modo en que son transmitidas, encontrando así vías de acceso directo (transmisión consciente y explicita de las habilidades emocionales) e indirecto (transmisión inconsciente e implícita) (Zeidner et al., 2003).

El empleo de una u otra vía va a venir determinado por el estilo educativo predominante en la familia, entendiendo los «estilos educativos parentales» como: esquemas prácticos que reducen las múltiples y minuciosas prácticas educativas paternas a unas pocas dimensiones, que, cruzadas entre sí en diferentes combinaciones, dan lugar a diversos tipos habituales de educación familiar (Torío, Peña y Rodrígez, 2008).

Según diversos autores (Feldman, 2007; Ochaita, 1995; Solé y Gallart, 1998) los modelos o estilos educativos se configuran a partir de cuatro aspectos distintos en las conductas de los progenitores: afecto en la relación, el grado de control, el grado de madurez y la comunicación entre padres-hijos. Estas dimensiones y su combinación procuran unas experiencias educativas diversas que los niños viven en su familia y que naturalmente influirán en su desarrollo. Son en estas relaciones familiares donde el niño aprende y pone en juego unas determinadas habilidades, como por ejemplo, el entusiasmo, el autocontrol, el reconocimiento de los propios sentimientos y el de los demás, entre otros.

Baumrind (1978), identificó tres estilos educativos parentales, mediante los cuales los padres controlan la conducta de sus hijos: a) estilo autoritario; b) permisivo; y c) un estilo que bautizó como autoritativo, siendo este último el que tendría mejores resultados. Así, los niños de padres autoritativos, comparados con los de padres autoritarios o permisivos, eran más maduros y competentes.

Partiendo de estos estudios, otros autores han venido ofreciendo otras clasificaciones (e.g., Alberdi, 1995; Hoffman, 1970; López. 1998; MacCoby y Martín, 1983; Ochaita, 1995; Torres, Alvira, Blanco y Sandi, 1994). Todas coinciden en afirmar que cada uno de los patrones educativos paternos produce diferencias en la conducta de los hijos. En consecuencia, el estilo educativo de los padres causará efectos distintos en función de las estrategias seleccionadas y también dependerán de la forma de los factores que pertenecen a lo que se conoce como inteligencia emocional.

Muchos estudios han investigado los efectos del estilo de crianza en el desarrollo emocional y el comportamiento de los hijos. El estilo parental predice la competencia socioemocioal de los hijos, y particularmente el estilo parental paterno se relaciona con las funciones psicosociales del hijo y con los sentimientos de soledad y depresión (McDowell, Parker \& Wang, 2003). Los estilos parentales autoritarios se han asociado a desordenes obsesivo-compulsivos (Timpano, Keough, Mahaffey, Schmidt y Abramowitz, 2010). Se ha tratado de asociar un estilo autoritativo (democrático) a una menor depresión en adolescentes (Liem, Cavell y Lustig, 2010). Por otro lado, estudios hechos con adolescentes disruptivos muestran que el estilo autoritario es el menos efectivo para mantener a estos chicos alejados de problemas (Pezzella, 2010). El estilo maternal permisivo se ha asociado al comportamiento antisocial de forma directa e indirecta, por su efecto en el desarrollo de la empatía de los hijos (Schaffer, Clark y Jeglic, 2009). El estilo parental cálido se ha asociado fuertemente con la inteligencia emocional de los adolescentes y en el caso de los chicos, el estilo parental paterno predice la autoestima de los hijos (Zukauskiené, Malinauskiené y Erentaité, 2011).

Estudios de revisión teórica, como el de Páez Campos, Zubieta y Casullo, (2009) y el de Cuervo (2010) o el de Alegre (2011). vinculan empíricamente una asociación positiva entre la inteligencia emocional (tanto la IE percibida como de rendimiento) y el apego seguro caracterizado por la presencia estable de cuidadores, vínculos parentales cálidos, cohesión y flexibilidad. Estos autores añaden que el apego seguro y la alta inteligencia emocional se asocian a maneras de afrontamiento más adaptativas y a una mejor salud mental, por lo que se puede deducir que el sentirse estimado, respetado y aceptado favorece la ejecución de conductas adaptativas y en resultado el número de actos antisociales se verían disminuidos. Por el contrario, un apego inseguro se correspondería a malas relaciones infantiles y a poca calidez en clima familiar, pues adultos evasivos le otorgan menos atención a la afectividad y a los hechos emocionales.

Por tanto, parecería fácil afirmar que padres emocionalmente inteligentes, cercanos y democráticos en sus prácticas pueden efectivamente transmitir sus habilidades emocionales a sus hijos (Alegre, 2012). Sin embargo, los estudios en edades tempranas son escasos y las medidas de evaluación utilizadas muy dispares, por lo que no contamos con suficiente información para dar una afirmación definitiva sobre el estado de la cuestión, sería necesaria una mayor investigación al respecto. 
El objetivo general de la investigación es profundizar en la incidencia que las características parentales tienen sobre la Inteligencia Emocional de los hijos en edades tempranas. Para acometer el objetivo general se plantean los siguientes objetivos específicos:

1. Identificar la relación de la IE de los padres con su estilo parental

2. Estudiar la relación del estilo parental y la IE de los padres y madres con la IE de los hijos.

2. a. Diferencia de medias en IE de los niños dependiendo del estilo parental paterno

2. b. Diferencia de medias en IE de los niños dependiendo del estilo parental materno

2. c. Relación de la IE de padres y madres con la IE de los hijos

3. Conocer los factores parentales (IE de los padres, estilo parental, edad, tiempo que pasan con sus hijos y la calidad de ese tiempo compartido) que pueden predecir la IE de los niños

\section{Método}

Para abordar nuestros objetivos de investigación se ha optado por elegir un diseño expo-facto, que trata de explicar la relación entre variables existentes, es una investigación basada sólo en la observación, Y puesto que el propósito principal es saber cómo se puede comportar un concepto o variable (la IE de los niños) conociendo el comportamiento de otra $\mathrm{u}$ otras variables relacionadas (estilos parentales e IE de los padres), sería un estudio correlacional.

Asimismo, la investigación será de corte cuantitativo y en ella se emplearán cuestionarios en la recogida de información, así como pruebas de habilidad en la percepción emocional de los niños.

\section{Participantes y contexto}

En el estudio participaron un total de 83 niños (39 niños y 44 niñas) escolarizados en cuatro centros distintos del noroeste de la Región de Murcia. Todos atendían a $2^{\circ}$ ciclo de Educación Infantil, concretamente al tercer nivel, de 5 años $(\mathrm{M}=4.95$ años, $\mathrm{DT}=21)$. De estos centros tres son de carácter público $(\mathrm{n}=39$ alumnos $)$ y uno es concertado ( $n=45$ alumnos).

Además, se solicitó la participación de padres y madres en este estudio. De los 120 permisos repartidos, 83 padres y 83 madres accedieron a participar en la investigación. Sin embargo, de ellos un total de 52 padres y 62 madres entregaron finalmente los cuestionarios cumplimentados, constituyendo así la muestra adulta de participantes.

La edad de las madres oscilaba entre 24 y 56 años (M $=36.70$ años, DT $=6.07)$, mientras que la edad de los padres oscilaba entre 28 y 68 años $(\mathrm{M}=39.7$ años, $\mathrm{DT}=$ 7.39). La mayoría de los participantes informaron estar casados ( $84.61 \%$ hombre, $80 \%$ mujeres) y tener una formación básica (25 padres y 19 madres), siendo escasos los padres que ostentan un título universitario (12 madres con diplomatura o licenciatura y 7 padres con diplomatura o licenciatura).

En cuanto al nivel socioeconómico, los padres reportan en la mayoría de los casos una economía suficiente, $26,95 \%$ de los 49 participantes (tanto padres como madres) declaran tener una economía suficiente, seguido por un $17,5 \%$ de economía limitada y un $3,92 \%$ opinaron que su economía era más que suficiente.

La selección de los participantes se realizó mediante un muestreo de carácter incidental, utilizando como muestra a los alumnos de centros a los que se tiene fácil acceso.

\section{Variables del estudio}

En cuanto a las variables de los participantes adultos, es decir de los padres y madres se recogen las variables de edad y sexo, sus estilos parentales y su inteligencia emocional. Todas estas variables tienen un carácter independiente en nuestra investigación, pues pretendemos ver el efecto de estas sobre la IE de los hijos.

En cuanto a las variables de los participantes infantiles (los niños), se han considerado las siguientes: edad, sexo y su inteligencia emocional. Las variables de sexo y edad serán independientes, mientras que la IE de los niños es la variable dependiente de este estudio. Para el estudio se realizó un análisis previo de sentencias para identificar los criterios de asignación de guarda y custodia que utilizaban los jueces en base a la estructura legal. A partir de ello, se utilizó un protocolo confeccionado por los autores, orientado a la exploración de los criterios de asignación de guarda y custodia. La variable independiente fue la introducción de la Ley $25 / 2010$, de 29 de Julio, del libro $2^{\circ}$ del Código Civil de Cataluña. Las variables dependientes fueron las sentencias judiciales emitidas entre enero de 2007 y junio de 2010 y entre julio de 2010 y diciembre de 2013. 


\section{Instrumentos}

Para la recogida de datos se han utilizado tres cuestionarios para los padres y una prueba de habilidad para los niños. Más específicamente, los instrumentos que se emplean para la recogida de información son:

\section{Para recogerinformación sobre los padres}

Cuestionario de características socio-demográficas:

Se trata de un cuestionario, diseñado para conocer aspectos del contexto familiar de los alumnos participantes (número de hermanos, horas que comparten padres e hijos, calidad de estas relaciones, etc.) y características de los padres (edad, ocupación, formación). De la información recogida, sólo una parte se utiliza en este estudio.

\section{Cuestionario sobre IE para adultos (padres)}

En este estudio se han utilizado 34 ítems extraídos del cuestionario original diseñado por Bar-On (1997). Dichos ítems fueron escogidos del manual del cuestionario original por tener las mayores cargas factoriales. Con el objetivo de estudiar las propiedades psicométricas del cuestionario se llevó a cabo un análisis de los ítems, el estudio de su estructura factorial, y de la fiabilidad de las dimensiones encontradas. No se hallaron las dimensiones propuestas por Bar-On, los ítems del cuestionario se agruparon en cuatro escalas, más la escala de estado de ánimo. Debido a la baja fiabilidad de las subescalas encontradas y al hecho de que algunas eran contradictorias entre sí, se decidió hallar un único factor de Inteligencia Emocional, cuya puntuación se computó utilizando el método de regresión, la fiabilidad encontrada para esta escala fue de $\alpha=.687$ para los 34 elementos que la componen..

\section{Cuestionario de estilos parentales}

En esta investigación se ha utilizado el cuestionario publicado por Comprehensive Psychological Assessment Centre (2009), el cual es una adaptación del cuestionario elaborado por Robinson, Mandleco, Olsen y Hart (1995). Este cuestionario está compuesto por 30 ítems y evalúan los siguientes estilos parentales: democrático (13 ítems), autoritario (13 ítems) y permisivo (4 ítems). Al estudiar las propiedades psicométricas del cuestionario en nuestra muestra $(n=114)$ se encontraron dos factores: 1) el estilo democrático ("con autoridad") cuya fiabilidad fue de $\alpha=.796$ y 2) el estilo autoritario, cuya fiabilidad fue de $\alpha=.820$. Quedando los ítems del estilo permisivo repartidos en los dos factores anteriores.
Se ha tomado como medida de la inteligencia emocional de los niños la información del EQ-360 $0^{\circ}$ ofrecida por los padres y madres de los alumnos participantes (hallándose una puntuación combinada de ambos observadores)

\section{Para la recogida de información sobre los alumnos:}

\section{Prueba de habilidad PERCERVAL (Mestre, et al. 2011)}

El PERCERVAL es la versión mejorada del PERVALEX (PERcepción, VALoración y EXpresión de emociones, Mestre, Guil, Martínez-Cabañas, Larrán y González, 2011). PERCERVAL es una prueba de 16 ítems elaborada con el fin de valorar la competencia socio-emocional de niños entre 3 y 6 años, concretamente la capacidad de percepción de las expresiones emocionales básicas (alegría, miedo, tristeza, enfado, asco y sorpresa), así como de la valoración de las mismas.

En esta prueba se les muestra a los niños diferentes expresiones emocionales en una presentación de powerpoint y han de indicar cuál de ellas corresponde con la emoción básica indicada. Hay dos tipos de presentaciones. La primera de ellas muestra una cuestión sobre qué expresión debería poner el personaje Alex ante determinadas situaciones de la vida cotidiana. Y el segundo tipo de diapositivas trata de evaluar situaciones más ambiguas para el niño, donde debe discriminar entre dos personajes (hermanas gemelas: Cris y Tina) cuál de los dos (o ninguna) es más probable que sienta una emoción específica. Es por tanto, una situación que requiere la percepción y después la valoración y esfuerzo del niño o niña para discernir cuál de las dos expresiones se ajusta más a la emoción planteada.

Dado que esta versión renovada no ha sido previamente utilizada, se ha optado por estudiar sus propiedades psicométricas. Cabe señalar que la prueba se compuso de dos factores: un primer componente formado por los ítems más sencillos, y un segundo componente formado por los ítems más complejos de la prueba. Ambos obtuvieron una adecuada fiabilidad ( $\alpha=.796$ y $\alpha=.82$, respectivamente). Además, se ha obtenido una puntuación total del PERCERVAL, la cual se extrajo a través de un análisis factorial de análisis principales de segundo orden, y cuya puntuación se calculó por regresión.

Inventario de Inteligencia Emocional de EQ-i:YV$360^{\circ}$ (Bar-On y Parker, 2000)

Se trata de un cuestionario para valorar la inteligencia emocional de los alumnos desde el punto de vista de un observador, en este caso los padres. Consta de 38 ítems que valoran las siguientes dimensiones: (1) Intrapersonal; (2) Interpersonal; (3) Manejo del Estrés; (4) Adaptabili- 
dad; (5) Estado de ánimo general. Además, los inventarios ofrecen una valoración sobre la inteligencia emocional total.

Se han hallado adecuadas propiedades psicométricas en términos del estudio de ítems, estructura factorial y fiabilidad de las dimensiones. Aparte de estos factores se ha calculado un factor general de segundo orden, al que hemos llamado "IE total de los hijos", las puntuaciones de este factor fueron computadas utilizando el método de regresión.

\section{Procedimiento}

La aplicación de los diferentes instrumentos se realizó de forma individual, en el caso de los padres, a quien se le hacía llegar los cuestionarios por medio de la tutora de los niños, respondían de forma autónoma en casa. En el caso de los niños de Educación Infantil, estos se ausentaban del aula durante un tiempo aproximado de 10 minutos por niño. Antes de que los alumnos comenzaran a realizar los test debíamos de cerciorarnos de que el niño/a había entendido cada una de las instrucciones. Tras las explicaciones, se aplicó el test PERCERVAL (Mestre, et al., 2011), que al ser un instrumento de rendimiento, y no de autopercepción, requería una sesión de evaluación administrada por personal cualificado.

Para analizar los datos se ha utilizado el programa estadístico SPSS (Stadistical Package Social Science, versión 20, IBM, 2011), llevando a cabo análisis de carácter descriptivo, correlacional y en ocasiones inferencial, incluyendo técnicas de tipo predictivo/explicativo a partir de la información obtenida.
En cuanto al agrupamiento de padres y madres según su estilo parental, se restó a la puntuación del estilo democrático la del estilo autoritario (= democrático - autoritario). Los que obtuvieron puntuaciones mayores que 1 se consideran de tendencia democrática, mientras que los que obtuvieron valores inferiores a -1 se consideran de tendencia autoritaria. El resto, aquellos entre el valor -1 y +1 , se consideran de estilo indefinido o neutro.

En cuanto al tratamiento de los datos perdidos, cabe destacar que estos se refieren principalmente a la población adulta, ya que no todos los padres y madres cumplimentaron los cuestionarios. Se decidió que se tomarían como participantes únicamente los 83 padres y 83 madres que accedieron a participar en la investigación.

\section{Resultados}

A continuación exponemos los resultados, atendiendo a los objetivos planteados:

\section{Correlación entre estilos parentales e IE de los ni- ños}

La Tabla 1, muestra las correlaciones entre las variables de los estilos parentales materno y paterno y la inteligencia emocional de los niños. Se aprecia que la dimensión de la IE de los niños que más se correlaciona con los estilos parentales es el manejo del estrés, la cual se correlaciona de forma negativa con los estilos autoritarios $(r=$ $-.46, p=.003, r=-.373, p=.018)$ de madre y padre res-

Tabla 1

Correlación entre las variables del estilo parental materno y paterno y la IE de los hijos

\begin{tabular}{|c|c|c|c|c|c|c|c|c|c|c|c|c|}
\hline & 1 & 2 & 3 & 4 & 5 & 6 & 7 & 8 & 9 & 10 & 11 & 12 \\
\hline 1 & 1 & & & & & & & & & & & \\
\hline 2 & -.097 & 1 & & & & & & & & & & \\
\hline 3 & $.495^{* *}$ & -.164 & 1 & & & & & & & & & \\
\hline 4 & -.360 & $.556^{* *}$ & .053 & 1 & & & & & & & & \\
\hline 5 & $.441^{* *}$ & -086 & .168 & -.277 & 1 & & & & & & & \\
\hline 6 & -.014 & .174 & -.050 & .282 & $-.261^{*}$ & 1 & & & & & & \\
\hline 7 & -.239 & .153 & .242 & 109 & $-.284^{*}$ & .073 & 1 & & & & & \\
\hline 8 & $.326^{*}$ & -.278 & $.365^{*}$ & -.209 & $.530^{* *}$ & $-.337^{*}$ & $-.363^{* *}$ & 1 & & & & \\
\hline 9 & .170 & $-.461^{* x}$ & $.409^{\prime \prime *}$ & $-.373^{x}$ & .194 & -.116 & -.025 & $.316^{*}$ & 1 & & & \\
\hline 10 & $.326^{*}$ & -.072 & .061 & -.097 & .154 & .018 & -.222 & .065 & -.022 & 1 & & \\
\hline 11 & -.043 & -.103 & .175 & .033 & .022 & -.075 & .035 & .155 & .232 & .000 & 1 & \\
\hline 12 & .148 & -.115 & .169 & -.027 & .104 & -.048 & -.098 & .159 & .168 & $.707^{\star \star}$ & $.707^{10 \times}$ & 1 \\
\hline
\end{tabular}

Nota. 1. Madre Democrático, 2: Madre Autoritario, 3: Padre Democrático, 4: Padre Autoritario, 5: EQ Hijo pym (puntuación sumada de la percepción de padres y madres sobre la IE de sus hijos) intrapersonal, 6: EQ Hijo pym interpersoal, 7: EQ Hijo pym Adaptabilidad, 8: EQ Hijo pym Estado de Ánimo, 9: EQ Hijo pym Manejo de estrés, 10 PERCERVAL (IE del hijo evaluada con prueba de habilidad) sencillas, 11: PERCEVAL complejas, 12: Total PERCEVAL. 
pectivamente; y de forma positiva con el estilo democrático del padre $(r=.409, p=.009)$. La otra dimensión de la IE de los niños que guarda especial relación con los estilos educativos es el estado de ánimo, el cual se relaciona de forma positiva con los estilos democráticos $(r=.326, p=.043, r=.365, p=.022$ para el estilo materno y paterno respectivamente). Además cabe señalar que el estilo democrático de la madre se correlaciona también con la habilidad intrapersonal de los niños $(r=$ $.441, p=.004)$ y con una percepción emocional incipiente del reconocimiento de emociones básicas $(r=.326, p$ $=.038$ ).

\section{2. a. Diferencia de medias en IE de los niños depen- diendo del estilo parental paterno}

Con el propósito de saber si los estilos parentales marcan una diferencia entre la IE de los niños, vamos a dividir la muestra según el estilo parental de los padres. Se han dividido a los padres entre aquellos tendentes al estilo democrático $(\mathrm{n}=9)$ aquellos tendentes al estilo autoritario $(\mathrm{n}=10)$ y aquellos sin tendencia significativa. Para ello se resto la nota democrática menos la autoritaria, así, los que salieron con valores mayores que 1 se consideraron de tendencia democrática, mientras que los que salieron con valores menores que - 1 se consideraron de tendencia autoritaria. El resto se consideran neutros.

A continuación, hemos calculado las puntuaciones medias en inteligencia emocional de los hijos de los tres tipos de padres (Tabla 2). Las puntuaciones de los tres grupos son bastante similares, salvo para las dimensiones de intrapersonal, interpersonal, estado de ánimo y manejo del estrés. Destacando en todas ellas, salvo en interpersonal, los niños de padres democráticos.

El ANOVA indicó diferencias estadísticamente significativas en las dimensiones de manejo del estrés $[\mathrm{F}(2$, $37)=11.246, p<.001]$ y estado de ánimo $[\mathrm{F}(2,36)=$ $4.184, p=.023]$. Los análisis post-hoc indicaron que esas diferencias se encontraban entre los tres grupos para la dimensión manejo del estrés, y sólo entre el grupo democrático vs. autoritario en la dimensión estado de ánimo.

Tabla 2

Estadísticos descriptivos de las puntuaciones de IE de los niños cuando se agrupan según el estilo parental del padre

\begin{tabular}{|c|c|c|c|c|c|c|c|c|c|c|}
\hline & \multicolumn{2}{|c|}{$\begin{array}{r}\begin{array}{r}\text { Democrático } \\
n=9\end{array} \\
\end{array}$} & \multicolumn{2}{|c|}{$\begin{array}{r}\begin{array}{r}\text { Indefinido } \\
n=21\end{array} \\
\end{array}$} & \multicolumn{2}{|c|}{$\begin{array}{r}\text { autoritario } \\
n=10\end{array}$} & \multicolumn{2}{|c|}{ total $n=40$} & \multirow{2}{*}{ ANOVA } & \multirow{2}{*}{ Post-hoc } \\
\hline & $\mathbf{M}$ & DT & M & DT & M & DT & $\mathbf{M}$ & DT & & \\
\hline $\begin{array}{l}\text { EQ.Hijo.pym } \\
\text { Intrapersonal }\end{array}$ & .983 & 1.871 & -.118 & 1.467 & -.738 & 2.164 & -.026 & 1.809 & $\begin{array}{r}\mathrm{F}(2,37)=2.357 \\
p=.109\end{array}$ & \\
\hline $\begin{array}{l}\text { EQ.Hijo.pym } \\
\text { Interpersonal }\end{array}$ & -.878 & 1.656 & .296 & 1.739 & .315 & 1.028 & .036 & 1.614 & $\begin{array}{r}\mathrm{F}(2,37)=1.954 \\
p=.156\end{array}$ & \\
\hline $\begin{array}{l}\text { EQ.Hijo.pym } \\
\text { Adaptabilidad }\end{array}$ & .231 & 2.210 & .129 & 1.628 & .046 & 1.121 & .131 & 1.629 & $\begin{array}{r}\mathrm{F}(2,37)=.029 \\
p=.971\end{array}$ & \\
\hline $\begin{array}{r}\text { EQ.Hijo.pym } \\
\text { Ánimo }\end{array}$ & 1.093 & 1.035 & .224 & 1.342 & -.910 & 1.991 & .111 & 1.602 & $\begin{array}{r}\mathrm{F}(2,36)=4.184 \\
p=.023\end{array}$ & Demo $>$ auto \\
\hline $\begin{array}{r}\text { EQ.Hijo.pym } \\
\text { Estrés }\end{array}$ & 1.559 & 1.048 & .186 & 1.424 & -1.419 & 1.507 & .093 & 1.696 & $\begin{array}{r}\mathrm{F}(2,37)=11.246 \\
p<.001\end{array}$ & $\begin{array}{r}\text { Dem. }>\text { Ind. }> \\
\text { Auto }\end{array}$ \\
\hline $\begin{array}{r}\text { PERCEVAL } \\
\text { sencillas }\end{array}$ & .097 & .945 & .209 & .492 & -.087 & .785 & .110 & .682 & $\begin{array}{r}\mathrm{F}(2,37)=.628 \\
p=.539\end{array}$ & \\
\hline $\begin{array}{r}\text { PERCEVAL } \\
\text { compj. }\end{array}$ & .151 & .883 & -.003 & 1.013 & -.295 & 1.055 & -.042 & .985 & $\begin{array}{r}\mathrm{F}(2,37)=.507 \\
p=.606\end{array}$ & \\
\hline Total PERCEVAL & .248 & 1.505 & .206 & 1.218 & -.382 & 1.168 & .068 & 1.269 & $\begin{array}{r}\mathrm{F}(2,37)=.837 \\
p=.441\end{array}$ & \\
\hline
\end{tabular}


Tabla 3

Estadísticos descriptivos de las puntuaciones de IE de los niños cuando se agrupan según el estilo parental de la madre

\begin{tabular}{|c|c|c|c|c|c|c|c|c|c|c|c|}
\hline & \multicolumn{2}{|c|}{$\begin{array}{r}\text { Democrático } \\
n=9\end{array}$} & \multicolumn{2}{|c|}{$\begin{array}{r}\text { Neutro } \\
n=26\end{array}$} & \multicolumn{2}{|c|}{$\begin{array}{r}\text { Autoritario } \\
n=5\end{array}$} & \multicolumn{2}{|r|}{ Total } & \multirow[t]{2}{*}{ ANOVA } & \multirow[t]{2}{*}{$p$} & \multirow{2}{*}{$\begin{array}{r}\text { Post- } \\
\text { hoc }\end{array}$} \\
\hline & $M$ & DT & $\mathrm{M}$ & DT & $\mathrm{M}$ & DT & $\mathrm{M}$ & DT & & & \\
\hline $\begin{array}{l}\text { EQ.Hijo.pym } \\
\text { Intrap. }\end{array}$ & .69 & 1.81 & -.08 & 1.66 & -.86 & .89 & -.01 & 1,66 & $F(2,37)=1,54$ & .227 & \\
\hline $\begin{array}{l}\text { EQ.Hijo.pym } \\
\text { Interp. }\end{array}$ & .75 & 1.91 & -.39 & 1.06 & 1.15 & 2.00 & .06 & 1,51 & $F(2,37)=3,90$ & .029 & $\begin{array}{l}\text { Dem. < } \\
\text { Autor.* }\end{array}$ \\
\hline $\begin{array}{l}\text { EQ.Hijo.pym } \\
\text { Adap. }\end{array}$ & -.58 & 2.23 & -.13 & 1.66 & .94 & 1.35 & -.10 & 1.78 & $F(2,37)=1.20$ & .311 & \\
\hline $\begin{array}{l}\text { EQ.Hijo.pym } \\
\text { Ánimo }\end{array}$ & .53 & 1.22 & -.10 & 1.56 & -1.40 & 2.21 & -.14 & 1.64 & $F(2,36)=2.30$ & .114 & \\
\hline $\begin{array}{l}\text { EQ.Hijo.pym } \\
\text { Estrés }\end{array}$ & 1.02 & 2.22 & -.29 & 1.37 & -1.59 & 1.39 & -.16 & 1.73 & $F(2,37)=4.55$ & .017 & $\begin{array}{r}\text { Dem. > } \\
\text { Autor. }\end{array}$ \\
\hline $\begin{array}{l}\text { PERCEVAL S } \\
\text { PERCEVAL C }\end{array}$ & $\begin{array}{l}.00 \\
.26\end{array}$ & $\begin{array}{l}.90 \\
.97\end{array}$ & $\begin{array}{r}.11 \\
-.04\end{array}$ & $\begin{array}{r}.68 \\
1.10 \\
\end{array}$ & $\begin{array}{r}-.64 \\
.47 \\
\end{array}$ & $\begin{array}{l}.90 \\
.69\end{array}$ & $\begin{array}{l}.00 \\
.09\end{array}$ & $\begin{array}{r}.78 \\
1.03 \\
\end{array}$ & $\begin{array}{l}F(2,38)=2.05 \\
F(2,38)=.670\end{array}$ & $\begin{array}{l}.142 \\
.518\end{array}$ & \\
\hline $\begin{array}{l}\text { Total } \\
\text { PERCEVAL }\end{array}$ & .26 & 1.57 & .07 & 1.44 & -.16 & 1.20 & .08 & 1.41 & $F(2,38)=.143$ & .867 & \\
\hline
\end{tabular}

Nota. $\left(^{*}\right)$ Diferencias marginales sig.7 entre demócratas y autoritarias.

2. b. Diferencia de medias en IE de los niños dependiendo del estilo parental materno

La Tabla 3 muestra los estadísticos descriptivos de las puntuaciones de los alumnos con madres democráticas, autoritarias y de estilo parental indefinido.

Se aprecia que los hijos de madres autoritarias presentan puntuaciones de IE por debajo de sus compañeros en las dimensiones interpersonal, estado de ánimo y manejo del estrés, así como en la habilidad de percepción y valoración de emociones indicada en el total de la prueba PERCERVAL. Estos mismos alumnos son quienes más puntúan en las dimensiones referidas las habilidades interpersonales y adaptabilidad.

Por su parte, los hijos de madres democráticas puntúan más alto en intrapersonal, estado de ánimo y mane- jo del estrés, así como en el total de la prueba PERCERVAL, mientras que son quienes más bajo puntúan en adaptabilidad.

Los niños de madres con estilo parental indefinido presentan puntuaciones equilibradas con respecto a sus compañeros, salvo en interpersonal, y las preguntas complejas de la prueba PERCERVAL donde presentan las puntuaciones más bajas.

Para comprobar si estas diferencias eran estadísticamente significativas se condujo un ANOVA, el cuál reveló diferencias significativas únicamente en las variables de habilidades interpersonales $[\mathrm{F}(2,37)=3.909, p=.029]$ y en manejo del estrés $[\mathrm{F}(2,37)=4.554, p=.017]$; en ambos casos, a favor de los niños con madres de estilo democrático en relación con los niños de madres con estilo autoritario.

Tabla 4

Correlaciones entre la IE de los padres y la IE de los hijos

\begin{tabular}{|c|c|c|c|c|c|c|c|c|c|c|c|}
\hline & 1. & 2. & 3. & 4. & 5. & 6. & 7. & 8. & 9. & 10. & 11. \\
\hline 1 & 1 & & & & & & & & & & \\
\hline 2 & $.616^{\pi x}$ & 1 & & & & & & & & & \\
\hline 3 & $.398^{* *}$ & $.428^{\star *}$ & 1 & & & & & & & & \\
\hline 4 & $.327^{\star}$ & $383^{*}$ & $.760^{* *}$ & 1 & & & & & & & \\
\hline 5 & -.039 & .000 & $-.525^{* x}$ & $-.261^{*}$ & 1 & & & & & & \\
\hline 6 & .073 & -.101 & $-.529^{\pi x}$ & $-.284^{\pi}$ & .073 & 1 & & & & & \\
\hline 7 & $.507^{\pi \pi}$ & $.490^{\pi \pi}$ & $.845^{\star x}$ & $.530^{\pi \pi}$ & $-.337^{\star}$ & $-.363^{\pi x}$ & 1 & & & & \\
\hline 8 & .228 & .304 & $.505^{\pi x}$ & .194 & -.116 & -.025 & $.316^{\pi}$ & 1 & & & \\
\hline 9 & .140 & -.040 & .170 & .104 & -.048 & -.098 & .159 & .168 & 1 & & \\
\hline 10 & .185 & .270 & .107 & .154 & .018 & -.222 & .065 & -.022 & $.707^{\star \star}$ & 1 & \\
\hline 11 & .038 & -.190 & .141 & .022 & -.075 & .035 & .155 & .232 & $.707^{\star \pi}$ & .000 & 1 \\
\hline
\end{tabular}

Nota. 1. IE Padres, 2: IE Madres, 3: EQ Hijo pym Total, 4: EQ Hijo pym Intrapersonal, 5: EQ Hijo pym interpersonal, 6: EQ Hijo pym Adaptabilidad, 7: EQ Hijo pym Estado de ánimo, 8: EQ Hijo pym Manejo de estrés, 9: IE Hijos PERCEVAL, 10: PERCEVAL sencillas, 11: PERCEVAL complejas. **. La correlación es significativa al nivel 0,01 (bilateral). *. La correlación es significante al nivel 0,05 (bilateral). 
Tabla 5

Coeficientes de regresión e para las variables entradas en el modelo de regresión para predecir la habilidad perceptiva de emociones de los hijos (PERCERVAL)

\begin{tabular}{lccccc}
\hline & B & Error típico & Beta & $t$ & Sig. \\
\hline (Constante) & -6.108 & 1.517 & & -4.027 & .001 \\
Edad padre & .177 & .040 & .708 & 4.368 & .000 \\
\hline
\end{tabular}

2. c. Relación de la IE de padres y madres con la IE delos hijos

Según muestra la Tabla 4, la IE de padres y madres correlaciona de forma significativa con la IE de los hijos cuando ésta es medida a través del EQ-i:YV-360 , pero no cuando se mide a través de la prueba de habilidad PERCEVAL.

Las correlaciones más elevadas aparecen entre la IE de los padres y el estado de ánimo de los hijos $(r=.505$, $p=.001$ y $r=.490, p=.001$ con la IE de padres y madres respectivamente). La correlación entre la IE de los padres y madres con la IE total de los hijos también es de magnitud moderada y estadísticamente significativa $(r=.398, p=.010$ y $r=.428, p=.005)$. Además, la IE de los padres correlaciona significativamente con la habilidad intrapersonal de los niños $(r=.327, p=.037 \mathrm{y}$ $r=.383, p=.012)$.

Como se ha comentado anteriormente, cuando se mide la IE de los niños a través de una prueba de rendimiento las correlaciones entre la IE de padres e hijos no son estadísticamente significativas.

\section{Predicción de la IE de los hijos según los factores parentales}

Se quiso comprobar si los estilos parentales y la IE de los padres predecían de alguna manera la IE de los niños. Para ello se han llevado a cabo análisis de regresión lineal con el método de pasos sucesivos.
En el primer análisis se introdujo como variable dependiente la puntuación total en Percepción emocional (medida con el PERCERVAL), y como variables independientes los estilos parentales materno y paterno y la inteligencia emocional de los padres y madres, así como otras características de padres y madres: edad, tiempo que pasan con sus hijos, y la calidad de dicho tiempo

El programa arrojó un modelo significativo en el que sólo entro la variable "edad del padre" como predictora de la habilidad para percibir emociones de los hijos $\left(\mathrm{R}^{2}\right.$ corregida $=.475, \mathrm{~F}(1,19)=19.078, p<.001)$. Según este modelo la edad de los padres por sí sola explicaría un $47 \%$ de la varianza de la habilidad para percibir emociones de los hijos. La Tabla 5 ofrece información sobre las variables introducidas en el modelo.

Se ha llevado a cabo el mismo tipo de análisis para predecir la IE total de los hijos valorada por los padres (EQ-i:YV), obteniendo dos modelos estadísticamente significativos.

En el primer modelo entró como variable predictora de la IE de los hijos la IE de las madres $\left(\mathrm{R}^{2}\right.$ corregida $=$ $.552, \mathrm{~F}(1,19)=25.639, p<.001)$, explicando esta un $55 \%$ de la varianza de la IE de los niños.

El segundo modelo, incluía además el estilo autoritario de los padres, e incrementaba en $10 \%$ la varianza explicada $\left(\mathrm{R}^{2}\right.$ corregida $=.653, \mathrm{~F}(2,18)=19,803, p<$ $.001)$. La Tabla 6 ofrece información adicional sobre las variables predictoras incluidas en el segundo modelo.

Tabla 6

Coeficientes de regresión e para las variables entradas en el modelo de regresión para predecir la IE de los hijos estimada por los padres (EQ-i:YV-360)

\begin{tabular}{|c|c|c|c|c|c|c|}
\hline & & B & Error típico & Beta & $\mathbf{t}$ & Sig. \\
\hline \multicolumn{7}{|c|}{ Modelo 1} \\
\hline 1 & (Constante) & -.125 & .155 & & -.807 & .430 \\
\hline 1 & IE Madres & .693 & .137 & .758 & 5.063 & .000 \\
\hline \multicolumn{7}{|c|}{ Modelo 2} \\
\hline & (Constante) & -.047 & 140 & & -.333 & .743 \\
\hline \multirow[t]{2}{*}{2} & IE Madres & .534 & .136 & .584 & 3.934 & .001 \\
\hline & Padre_estilo_autoritario & -.386 & 151 & -.379 & -2.553 & .020 \\
\hline
\end{tabular}




\section{Discusión}

Los resultados de este estudio apuntan a que las variables de la IE de los niños más afectadas por los estilos parentales son el manejo del estrés y el estado de ánimo, obteniendo mejores niveles cuando los padres y madres tienden al estilo democrático.

Estos resultados, están de acuerdo con las conclusiones a las que llega Páez et al. (2006) quienes indican que un cuidador sensible a las señales del infante y que responde a ellas, características del padre democrático, inducirá una alta IE en los niños, es decir, una buena capacidad de regulación emocional, basada en la minimización del estrés, aceptación, etc. Esto coincide con el estudio de Baumrind (1978), quien estudió la conducta en niños de entre ocho y nuevos años y observó que los niños de familias democráticas tenían elevadas competencias sociales y de afrontamiento, en comparación con los otros estilos parentales.

Más concretamente, en nuestra investigación, el estilo democrático de la madre se correlaciona con la habilidad intrapersonal de los niños. Pensamos que esto se debe a quelas madres caracterizados por un estilo democrático suelen dar dos o más opciones de elección a los niños. Para estos niños, que tienen la oportunidad de elegir y por tanto, la oportunidad de acertar o equivocarse en su elección, podría ser más importante el conocerse a uno mismo para saber qué quieren en realidad y qué puede satisfacerlos entre dos opciones dadas.

El estilo democrático de la madre también correlacionó con una percepción emocional incipiente del reconocimiento de emociones básicas, quizás dicha relación se deba a que estos son niños que tienden a hablar con sus madres sobre su estado de ánimo, compartiendo emociones y dialogando acerca de las mismas. Este resultado recuerda a los resultados de Schaffer, Clark, y Jeglic, (2009), quienes evidenciaron que el estilo maternal influencia el desarrollo de la empatía en los hijos. La investigación previa apunta a que principalmente en el reconocimiento de expresiones faciales, observaron que la calidez y el sustento emocional parental, incluyendo las practicas de crianza más específicamente emocionales (como compartir emociones, hablar sobre ellas, su intensidad y frecuencia de aparición en las interacciones sociales), se relacionan positivamente con el conocimiento emocional de los niños (Bennett, Bendersky y Lewis, 2005).

En cuanto a los datos que indican una mejor habilidad interpersonal en hijos de madres autoritarias debemos decir que no encuentra suficiente consistencia ni apoyo en la literatura revisada, sin embargo, si se reduce el estilo autoritario a altos niveles de control parental encontramos que Mayer, Caruso y Salovey (1999) hablan de la necesidad de un alto control familiar en los hijos varones para un buen desarrollo de la empatía. Liau et al., (2003) también vincula el control parental a la inteligencia emocional. Sin embargo, otras investigaciones sugieren que el estilo parental democrático no está siempre asociado con los resultados más óptimos en niños y adolescentes (Kim y Rohner, 2002). De acuerdo con esta idea, estilos parentales similares tienen diferentes repercusiones en el ajuste de los niños dependiendo del entorno cultural en el que tiene lugar la socialización.

También se señala la existente relación entre la IE de los padres y la de los hijos, se muestra cómo la propia capacidad emocional de los padres influirá en el desarrollo emocional de sus hijos. Estos resultados coinciden con estudios como los de Palomera (2009) quien defiende que para adquirir habilidades emocionales es necesario contar con modelos emocionalmente habilidosos, es decir, con alta IE.

Además, se observa que la IE padres-hijos correlaciona mejor cuando la IE se mide en ambos casos con cuestionarios. Probablemente si hubiéramos medido la IE de de los padres con pruebas de habilidad, puede que ésta correlacionara de forma significativa con las puntuaciones obtenidas por los alumnos en la prueba de habilidad emocional (la medida PERCERVAL).

Sánchez Nuñez (2008) obtuvo resultados similares en su investigación, donde existe una clara relación entre la IE autoinformada de los padres (padre-madre) y la de sus hijos/as, medida a través del MEIS. En concreto, a mayor percepción de Claridad emocional por parte de los padres (padre-madre), mayor percepción de Claridad en sus hijos/as.

Guastello y Guastello (2003) estudiaron la relación entre la IE de padres/ madres e hijos/as a través de una escala de autoinforme, donde únicamente la IEA de la madre correlacionaba de forma significativa con la IEA de sus hijos/as, coincidiendo así con uno de los predictores de la IE de los niños que hemos encontrado en este estudio: la IE de la madre. En cambio, las otras características predictoras resultantes (edad y autoridad del padre) no se sostienen con investigaciones anteriores. Ambos resultados podrían ser atribuidos principalmente a factores socioculturales, de los cuáles sería interesante se ocuparan estudios posteriores.

En definitiva, nuestros resultados nos llevaría a asumir que padres emocionalmente inteligentes se relacionan de forma positiva con un estilo de crianza democrático, y éste a su vez con mayor regulación y percepción emocio- 
nal, así como, mejor estado de ánimo y manejo de estrés en niños, tal como sugerían las revisiones de la literatura (Alegre, 2011; Cuervo, 2010; Páez et al., 2006). Se puede concluir que padres inteligentes emocionalmente tienden a tener hijos con altos niveles de Inteligencia emocional.

Consideramos, pues, que este trabajo aporta evidencia empírica importante en el estudio de la relación entre los estilos educativos familiares y la autoestima del niño pequeño, y contribuye además a valorar la necesidad de estudiar los primeros entornos sociales como fuentes predictoras de la incipiente configuración del autoconcepto y del nivel de autoestima del niño pequeño. Evidenciándose la importancia del contexto familiar en el desarrollo de la regulación emocional de los niños (Morris, Silk, Stenberg, Myers y Robinson, 2007).

Pese a todo, hemos de ser precavidos a la hora de generalizar estos resultados y señalar ciertas limitaciones, ya que, la muestra de participantes ha sido pequeña y poco heterogénea. Además, se ha dejando a un lado la investigación de corte cualitativo, que hubiera podido aportar riqueza descriptiva, a lo que se suma la falta de literatura científica sobre el tema, lo que se puede explicar por varias razones: por una lado, la complejidad metodológica que conlleva la realización de estudios longitudinales y multivariados; por otro lado, por la dificultad que entraña la evaluación infantil en sí misma, unido a la falta de instrumentos de medición fiables de los procesos emocionales y en concreto de la IE infantil, donde las pruebas estandarizadas son muy escasas y la mayor parte no buscan un acceso directo al niño, sino que recogen información a través de terceros.

Nuestras principales propuestas de investigación futura se centrarían en dar continuidad al estudio, ampliando la muestra, proponiendo otras pruebas más fiables y planteando la conveniencia de seguir realizando estudios comparativos padres-hijos, y comprobar si se dan procesos bidireccionales en los efectos sobre ambos.

En cuanto a las implicaciones prácticas socioeducativas, consideramos necesaria la implementación de programas de educación emocional para padres, y así posibilitar una intervención más eficaz en el marco familiar con efectos positivos en el desarrollo personal de sus hijos (Nastasa y Sala, 2012) Esperamos que, a través de escuelas de padres o programas de educación emocional, se faciliten la adecuación entre las primeras figuras de crianza y los niños pequeños, se desarrollen conductas positivas, relaciones sociales satisfactorias, pudiendo ser útil para el éxito emocional de los hijos.. En el caso de los niños sería interesante integrar la Educación Emocional en el currículum de Educación Infantil, tal y como defienden Guil, Mestre, González y Foncubierta (2011). En este sentido, son varios los autores que coinciden en afirmar que la inteligencia emocional y la educación emocional, deben ser difundidas a través de la educación, con el objetivo de desarrollar competencias básicas para la vida que permitan alcanzar un mejor bienestar (Bisquerra, 2011). 


\section{Referencias}

Alberdi, I. (1995). Informe sobre la situación de la familia española. Madrid, España: Ministerio de Asuntos Sociales.

Alegre, A. (2011). Parenting Styles and Children's emotional intelligence: What do we know? The family Journal: Counseling and therapy for couples and families, 19(1) 56-62.

Alegre, A. (2012). The relation between the time mothers and children spent together and the children's trait emotional intelligence. Springer Science Business Media, LLC 2012.

Bar-On, R. y Parker, J. D. (2000). EQ-i: YV. Baron Emotional Quotient Inventory: Youth Version. Technical Manual. New York: MHS.

Bar-On, R. (1997). The Emotional Quotient Inventory (EQ-i): A test of emotional intelligence. Toronto, Canadá: Multi-Health Systems.

Baumrind, D. (1978). Parental disciplinary patterns and social competence in children, Youth and Society, 9, 239-276.

Bennett, D. S., Bendersky, M. y Lewis, M. (2005). Antecedents of emotion knowledge: Predictors of individual differences in young children. Cognition and Emotion, 19, 375-396.

Bisquerra, R. (Coord.). (2011). Educación emocional. Propuestas para educadores y familias. Bilbao, España: Desclée de brouwer.

Comprehensive Psychological Assessment Centre (CPAC). (2009). Parental Styles Questionnaire. Published online. Recuperado de http://www.comprehensivepsychology.com.au/assets /pdf/PARENTING\%20STYLE\%20QUESTIONNAI RE.pdf

Cuervo, A. (2010). Pautas de crianza y desarrollo socio afectivo en la infancia. Diversitas: Perspectivas en Psicología, 6(1), 111-121.

Eisenberg, N., Cumberland, A. y Spinrad, T. L. (1998). Parental socialization of emotion. Psychological Inquiry, 9, 241-273.

Feldman, R. S. (2007). Desarrollo psicológico a través de la vida. México: Pearson.
Ferrándiz, C., Fernández, C. y Bermejo, R. (2011). Desarrollo afectivo emocional y de la personalidad. En Prieto, M. D. (Coord.), Módulo formación básica. Psicología de la educación y del desarrollo infantil. (pp. 129-156). Valencia, España: Valencia International University.

Fúlquez, S. C. (2011). La inteligencia emocional y el ajuste psicológico: un estudio transcultural. (Tesis doctoral). Recuperado de la base de datos TESEO.

Goleman, D. (1996). Inteligencia emocional. Barcelona, España: Kairós.

Guastello, D. D. y Guastello, S. J. (2003). Androgyny, Gender Role Behavior, and Emotional Intelligence among college students and their parents. Sex Roles, $49,663-673$

Guil, R., Mestre, J. M., González, G. y Foncubierta, S. (2011). Integración del desarrollo de competencias emocionales en el currículum de Educación Infantil. REIFOP, 14 (3).

Hoffman, M. L. (1970) Conscience, personality and socialization techniques, Human Development, 13, 90126.

IBM Corp. (2011). IBM SPSS Statistics for Windows, Version 20.0. Armonk, NY: IBM Corp.

Jiménez-Morales, M y López-Zafra, E. (2009). Inteligencia emocional y rendimiento escolar: estado actual de la cuestión. Revista Latinoamericana de Psicología, 41(1), 69-79.

Kim, K. y Rohner, R. P. (2002). Parental warmth, control, and involvement in schooling: Predicting academic achievement among Korean American adolescents. Journal of Cross-Cultural Psychology, 33, 127140 .

León-Rodríguez, D. y Sierra, H. (2008). Desarrollo de la comprensión de las consecuencias de las emociones. Revista Latinoamericana de Psicología, 40(1), 35-45.

Liau, A. K., Liau, A. W. L., Teoh, G. B. S. y Liau, M. T. L. (2003). The case for emotional literacy: The influence of emotional intelligence on problem abehaviours in Malaysian secondary school students. Journal of Moral Education, 32, 51-66.

Liem, J. H., Cavell, E. C. y Lustig, K. (2010). The influence of authoritative parenting during adolescence on 
depressive symptoms in young adulthood: Examining the mediating roles of self-development and peer support. The Journal of Genetic Psychology, 171, 73-92.

López, E. (1998). La familia, nuevo contexto educativo ante el conflicto y la esperanza. Revista Complutense de Educación, 9(2), 79-100.

Maccoby, E. E. y Martin, J. A. (1983). Socialization in the context of the family: parent-child interaction. Handbook of Child Psychology, 4, 1-102.

Mayer, J. D., Caruso, D. y Salovey, P. (1999). Emotional intelligence meets tradicional standards for an intelligence. Intelligence, 27, 267-298.

Mayer, J. D. y Salovey, P. (1997). What is emotional intelligence? En P. Salovey y D. Sluyter (Eds.), Emotional Development and Emotional Intelligence: Implications for Educators (pp. 3-31). Nueva York: Basic Books.

McDowell, D., Parker, R. y Wang, S. (2003). Differences between mothers and fathers advice-giving style and content: Relations with social competence and psychological functioning in middle childhood. Merrill-Palmer Quarterly, 49 (1), 55-76.

Mestre, J. M., Guil, R., Martínez-Cabañas, F., Larrán, C. y González, G. (2011). Validación de una prueba para evaluar la capacidad de percibir, expresar y valorar emociones en niños de la etapa infantil. REIFOP, 14 (3).

Morris, A., Silk, J., Stenberg, L., Myers, S. y Robinson, L. (2007). The Role of the family context in the development of emotion regulation. Social Develoment, 16(2), 362-388.

Nastasa, L. E. y Sala, K. (2012). Adolescents' emotional intelligence and parental styles. Procedia - Social and Behavioral Sciences, 33 (2012), 478-482.

Ochaita, E. (1995) Desarrollo de las relaciones padres/hijos. Infancia y Sociedad, 30, 206-227

Páez, D., Fernández, I., Campos, M., Zubieta, E. y Casullo, M. (2006). Apego seguro, vínculos parentales, clima familiar e inteligencia emocional: socialización, regulación y bienestar. Ansiedad y Estrés, 12 (2-3), 329-341.

Palomera, R. (2009). El posible papel del contexto familiar en el desarrollo de la inteligencia emocional. En
P. Fernández-Berrocal, N. Extremera, R. Palomera, D. Ruiz-Aranda, J. M. Salguedo y R. Cabello (Coords.), Avances en el estudio de la inteligencia emocional. I Congreso Internacional de Inteligencia Emocional (pp. 457-461). Santander, España: Fundación Marcelino Botín.

Pezzella, F. S. (2010). Authoritarian parenting: A race socialization protective factor that deters African American adolescents from delinquency and violence (Doctoral dissertation). Recuperado de la base de datos ProQuest Dissertations and Theses database. (UMI No. 3398173)

Robinson, C., Mandleco, B., Olsen, S. F. y Hart, C. H. (1995). Authoritative, authoritarian, and permissive parenting practices: Development of a new measure. Psychological Reports, 77, 819-830.

Sánchez Núñez, M. T. (2008). Inteligencia emocional autoinformada y ajuste perceptivo en la familia. Su relación con el clima familiar y la salud mental. (Tesis doctoral). Universidad de Catilla-La Mancha, Cuenca.

Schaffer, M., Clark, S. y Jeglic, E. L. (2009). The role of empathy and parenting style in the development of antisocial behaviors. Crime \& Delinquency, 55, 586599. doi: 10. 1177/0011128708321359

Schore, A. N. (1996). The experience-dependent maduration of a regulatory system in the orbital prefrontal cortex and the origin of developmental psychopathology. Developmentand Psychopathology, 8(1), 59-87.

Solé I. y Gallart, I. (1998) Las prácticas educativas como contextos de desarrollo. En C. Coll (Coord.), Psicología de la educación (pp. 137-216). Barcelona, España: Edhasa.

Timpano, K. R., Keough, M. E., Mahaffey, B., Schmidt, N. B. y Abramowitz, J. (2010). Parenting and obsessive compulsive symptoms: Implications of authoritarian parenting. Journal of Cognitive Psychotherapy: An International Quarterly, 24(3), 151-164. doi: 10. 1891/0889-8391.24.3.151

Torío, S., Peña, J. V. y Rodrígez, M. C. (2008). Estilos educativos parentales. Revisión bibliográfica y reformulación teórica. Teoría de la Educación, 20, 151178.

Torres, M., Alvira, F., Blanco, F. y Sandi, M. (1994). Relaciones padres/hijos. Madrid, España: Ministerio de Asuntos Sociales. 
Zeidner, M., Matthews, G., Roberts, R. D. y MacCann, C. (2003). Development of Emotional Intelligence: towards a multi-level investment model. Human Development, 46, 69-96.

Zukauskiené, R., Malinauskiené, O. y Erentaité, R. (2011). Effects of parenting styles and emotional intelligence on self-efficacy and self-esteem in late adolescence: gender differences. Psichologija, Journal of Vilnus University, 44, 22-41. 